\title{
Early Experience with ASDL in lcc
}

\author{
DAVID R. HANSON \\ Microsoft Research, 1 Microsoft Way, Redmond, WA 98052 USA \\ (email:drh@microsoft.com)
}

\begin{abstract}
SUMMARY
The Abstract Syntax Description Language (ASDL) is a language for specifying the tree data structures often found in compiler intermediate representations. The ASDL generator reads an ASDL specification and generates code to construct, read and write instances of the trees specified. Using ASDL permits a compiler to be decomposed into semi-independent components that communicate by reading and writing trees. Each component can be written in a different language, because the ASDL generator can emit code in several languages, and the files written by ASDL-generated code are machine- and language-independent. ASDL is part of the National Compiler Infrastructure project, which seeks to reduce dramatically the overhead of computer systems research by making it much easier to build high-quality compilers. This paper describes dividing lcc, a widely used retargetable $\mathrm{C}$ compiler, into two components that communicate via trees defined in ASDL. As the first use of ASDL in a 'real' compiler, this experience reveals much about the effort required to retrofit an existing compiler to use ASDL, the overheads involved, and the strengths and weaknesses of ASDL itself and, secondarily, of lcc. Copyright $\odot 1999$ John Wiley \& Sons, Ltd.
\end{abstract}

KEY WORDS: compilers; compiler infrastructure; intermediate representations; abstract syntax trees; lcc

\section{INTRODUCTION}

High-quality compilers for a range of modern languages are essential for conducting experimental research in computer architecture, programming languages, and programming environments. For example, compilers are required to run benchmarks for evaluating new ideas in architecture and code optimization. And compilers for new languages need optimizers, code generators and runtime systems for existing platforms.

Building compilers is often a bottleneck in these kinds of research projects because compiler construction is a labor-intensive activity. Often, nearly complete compilers must be constructed even if the essential components are relatively small parts of the whole. To evaluate a new architecture, for instance, requires a code generator for that architecture and perhaps an architecture-dependent optimizer. Writing toy compilers for toy languages is insufficient: The research community demands measurements using established benchmarks, like the SPEC benchmarks [1], which are written in real programming languages.

The National Compiler Infrastructure (NCI) project seeks to reduce dramatically the effort needed to perform realistic experiments by making it much easier to build high-quality compilers. The goal is to make it possible to build complete compilation systems from pieces, replacing or modifying only those components that are relevant to the client researchers. For

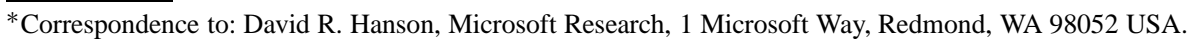

CCC 0038-0644/99/050417-19\$17.50

Copyright $\odot 1999$ John Wiley \& Sons, Ltd.
Received 8 October 1998

Revised 18 December 1998 Accepted 18 December 1998 


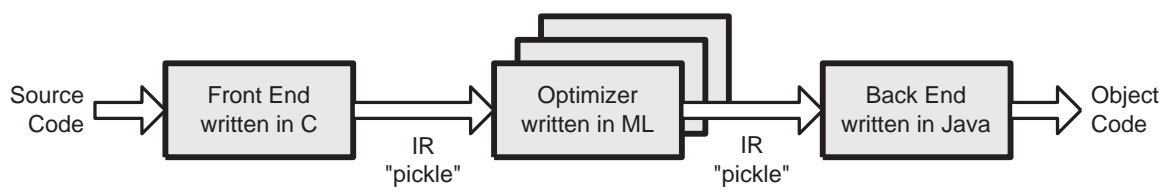

Figure 1. Sample compiler organization using ASDL

example, researchers studying global optimization algorithms for $\mathrm{C}++$ would replace or add only their optimizers and would use existing $\mathrm{C}++$ front ends and code generators.

The NCI can pay both economic and intellectual dividends. It should reduce significantly the costs of doing computer systems research, in terms of time, barrier to entry, and direct monetary outlay. It should also encourage more researchers to attack computer systems problems and thus increase the rate at which new research results appear.

The NCI includes the Stanford University Intermediate Format [2] and the emerging Zephyr program-generation tools, which includes the Abstract Syntax Description Language (ASDL) [3]. ASDL describes the abstract syntax of compiler intermediate representations and other tree-like data structures. The ASDL generator, asdlGen, converts ASDL specifications into appropriate data-structure definitions, constructors, and functions to read and write these data structures to files in a variety of programming languages.

This paper describes how ASDL is used with lcc [4], a well-documented, small, productionquality compiler for ISO Standard C [5]. This experience is valuable for two reasons. First, lcc is perhaps the simplest $\mathrm{C}$ compiler available and thus provides a 'basis' test case for ASDL and other NCI tools. If ASDL can't handle lcc's intermediate representation, it's unlikely to work in more ambitious compilers or in compilers for higher-level languages. Secondly, lcc wasn't designed to be decomposed into reusable program components, so doing so suggests how difficult it is to retrofit ASDL into existing compilers.

\section{ASDL}

ASDL is a small, domain-specific language for describing tree data structures [3]. ASDL specifications are concise and independent of any particular programming language. The ASDL generator, asdlGen, accepts an ASDL specification and emits code that defines a concrete representation for the data structures described in the specification, along with code that constructs, reads, and writes instances of those data structures. Currently, asdlGen can emit data-structure implementations in $\mathrm{C}, \mathrm{C}++$, Java, ML, and Haskell. ASDL specifications tend to be much smaller than the corresponding language-specific data-structure and function definitions.

Compiler writers can use ASDL to partition a compiler into several independent programs, as depicted in Figure 1. A front end reads source code and builds an intermediate representation (IR) using the data-structure constructors generated by asdlGen. It writes these data structures to a file- $\mathrm{a}$ 'pickle' - using the I/O functions generated by asdlGen. Subsequent phases read and write pickles as necessary, perhaps modifying them in the process. For example, optimizers would read a pickle, improve the code therein, and write a new pickle.

The binary pickle format is independent of both language and host platform. Thus, as suggested in Figure 1, compiler phases can be written in whatever ASDL-supported language best suits the task at hand. If ASDL becomes widely used, researchers can tap into a complete compiler by adding new phases or replacing just the phases of interest. 
ASDL is not a universal intermediate representation [6], because it supports any IR that can be described by trees. Likewise, ASDL is not a universal distribution format [7], because it does not mandate specific formats, capabilities, or platforms. ASDL and asdlGen are to compilers what interface definition languages (IDL) [8] and stub generators are to distributed systems. Typical IDLs describe the interfaces between program components running in different address spaces, and stub generators generate implementations of these functions that use remote procedure calls to communicate between clients and servers. ASDL describes the data-structure interfaces between compiler phases, and asdlGen generates functions to communicate between these phases.

ASDL is such a simple language that examples suffice to explain nearly all of its features. The following ASDL specification describes an IR for a language of arithmetic expressions, assignment statements, and print statements.

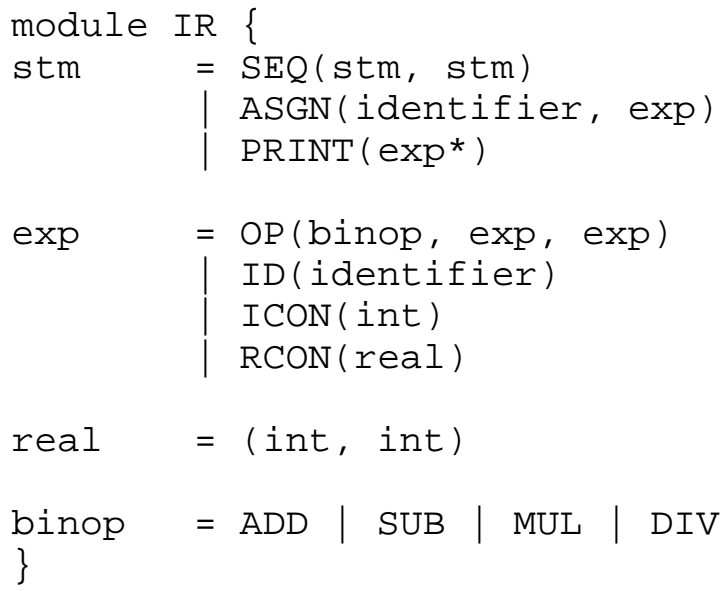

This specification defines four types: stm, exp, real and binop. The first three productions define the sum type stm, which has three constructors. A stm is a SEQ tree with two stm children, an ASGN tree with two children of types identifier and exp, or a PRINT tree with one child of type list of exp. identifier is a built-in type, and the ' $*$ ' following a type specifies a list of that type.

Similarly, exp is a sum type with four constructors that describe trees for binary operators, identifiers, and integer and real constants. int is another built-in type, but there is no built-in type for reals. So, the real type is a product type whose instances represent real numbers as two integers. Finally, bi nop is a simple sum type that defines constructors for each of the possible binary operators.

All four types are wrapped in a module named IR; this name is used to provide a disambiguating prefix for the names in the generated implementations.

It is easy to confuse ASDL specifications with grammars for programming languages. This ASDL specification describes the abstract syntax of the intermediate representation for programs written in some unspecified concrete syntax.

Given an ASDL specification, asdlGen emits an interface and an implementation in the programming language specified. The interface defines the concrete, language-specific representation for the types and declares functions for constructing instances of those types and for reading and writing them. The functions themselves appear in the implementation. For languages that do not separate interfaces and implementations, like Java, asdlGen emits a single implementation. 


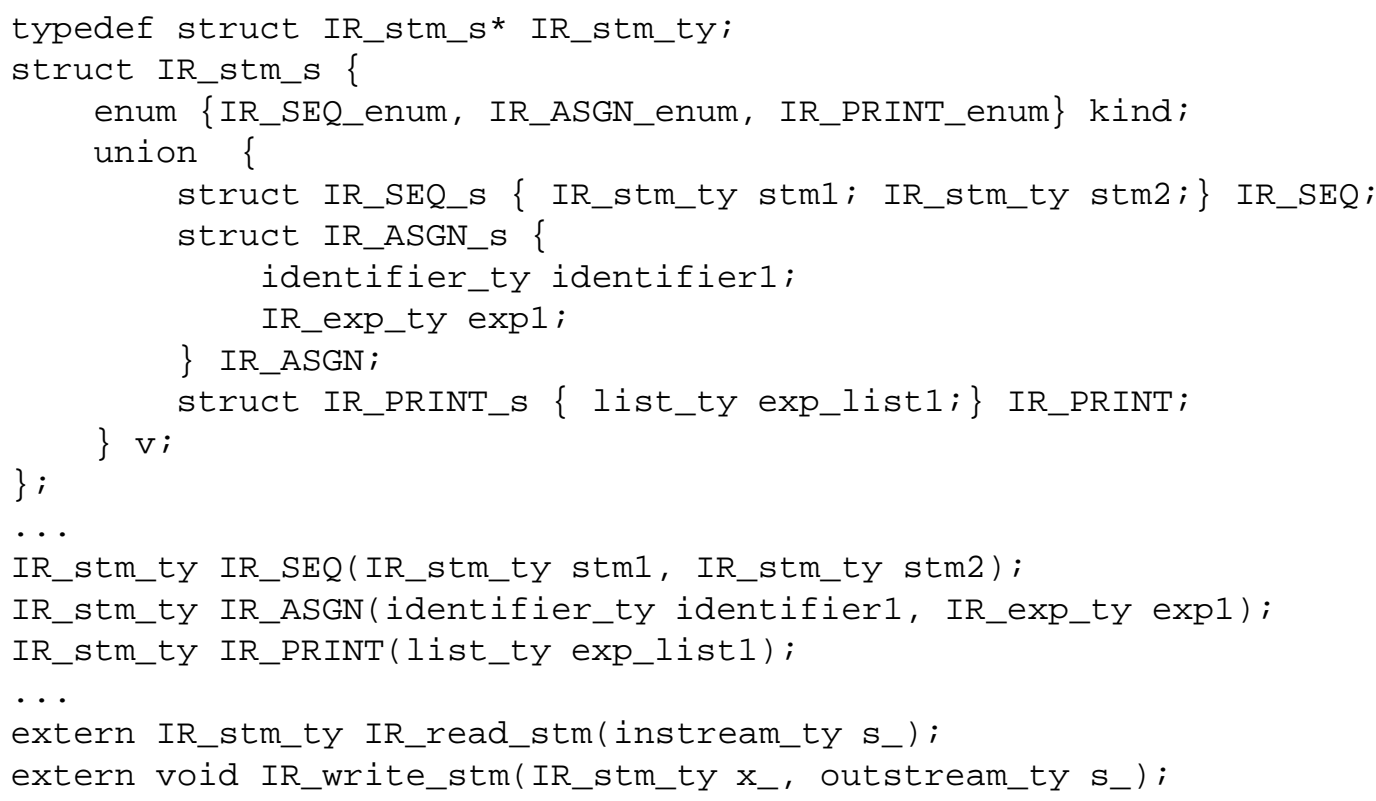

Figure 2. Generated C interface for the example ASDL specification

In $\mathrm{C}$, for example, given the ASDL specification above in the file IR . as d l, asdlGen writes the interface to IR. $h$ and the implementation to IR. C. Figure 2 shows the snippets from IR.h that define the representation for stm and the associated constructors, readers and writers. ASDL uses compact and efficient representations whenever possible. A sum type is represented by a union. There is one field for each constructor and a corresponding function for building instances of that constructor, as shown for stm in Figure 2. ASDL represents simple sum types with integers or their language-specific equivalent. In $\mathrm{C}$, for example, binop is represented by just an enumeration type. The implementation, IR . C, contains the definitions for the functions declared in IR.h. For example, the constructor IR_SEQ is

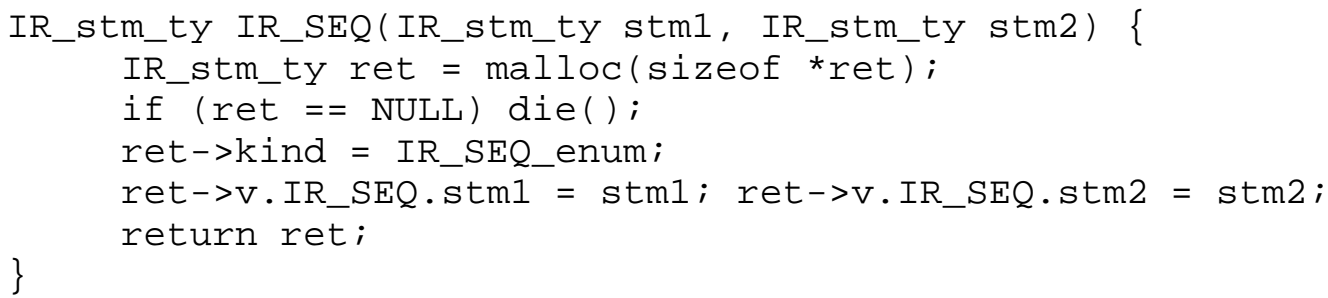

asdlGen generates constructors, but not deallocation functions; in languages without automatic storage management, clients must do explicit deallocations, when necessary.

ASDL comes with libraries of basic types and functions for each programming language it supports. These libraries provide support for polymorphic lists and for the built-in types, such as identifier, in languages that do not support them directly. In C, lists are represented by an implementation of variable-length sequences [9] (Ch. 11), and identifiers are represented by a $\mathrm{C}$ implementation of atoms [9] (Ch. 3). 
As Figure 2 reveals, asdlGen generates field names and parameter names as necessary to complete the data structure and function definitions. Except for the constructors, readers and writers, asdlGen does not provide additional functions to manipulate the data structures defined in a grammar; client code must manipulate them explicitly by referencing the appropriate fields. So, grammar writers can specify the field names in the ASDL specification. For example, if stm is defined as

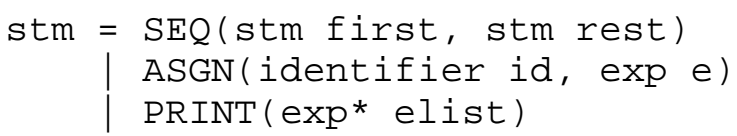

first, rest, id, e and elist will be used for the corresponding field and parameter names in Figure 2.

Sum types can also have attributes, which are fields that are common to all constructors. For example,

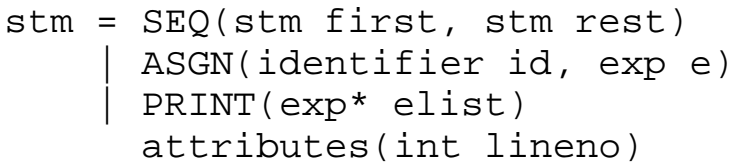

attaches a line number attribute to each constructor. Attributes are usually factored into a common prefix for the type, e.g. the $\mathrm{C}$ type for stm from Figure 2 becomes

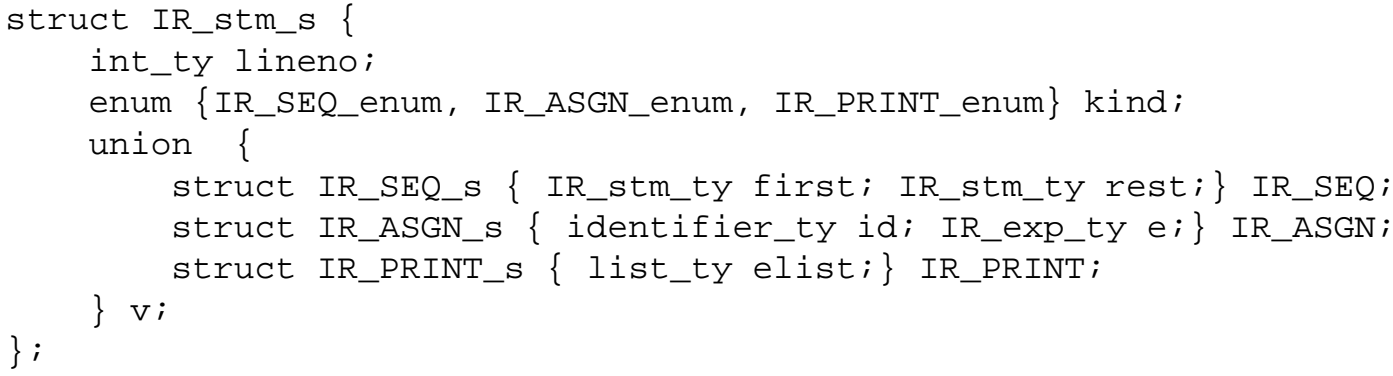

\section{THE lcc CODE-GENERATION INTERFACE}

lcc is a retargetable compiler for ISO Standard C. It is distributed with back ends for the SPARC, MIPS, X86 and ALPHA for several platforms. Others have written back ends for additional platforms, and lcc is used by other compiler researchers; for example, a modified, older release of lcc is used as the C compiler in the SUIF project.

Communication between lcc's target-independent front end and its target-dependent back ends is specified by a small code-generation interface. This interface consists of a few shared data structures, a 33-operator tree IR that represents executable code, and 18 procedures that manipulate and modify trees and the shared data structures.

The shared data structures include tree nodes, symbol-table entries, and types. The 33 tree IR operators are listed in Figure 3. Each of these generic operators can be specialized by appending an operand type suffix and a size in bytes. The six type suffixes are:

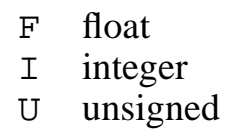




$\begin{array}{lllllllll}\text { CNST } & \text { ARG } & \text { ASGN } & \text { INDIR } & \text { CVF } & \text { CVI } & \text { CVP } & \text { CVU } & \\ \text { NEG } & \text { CALL } & \text { RET } & \text { ADDRG } & \text { ADDRF } & \text { ADDRL } & \text { ADD } & \text { SUB } & \\ \text { LSH } & \text { MOD } & \text { RSH } & \text { BAND } & \text { BCOM } & \text { BOR } & \text { BXOR } & \text { DIV } & \\ \text { MUL } & \text { EQ } & \text { GE } & \text { GT } & \text { LE } & \text { LT } & \text { NE } & \text { JUMP } & \text { LABEL }\end{array}$

Figure 3. lcc tree IR generic operators.

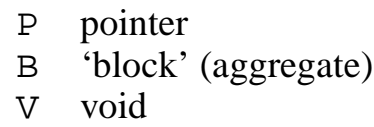

There can be up to 9 sizes. For example, ADDF 4 denotes a 4-byte floating addition, and CVII2 denotes a conversion from an integer to a 2-byte integer. While it looks like there are $33 \times 6 \times 9=1782$ specific operators, not all combinations are meaningful, and the number of sizes on most targets is limited. On 32-bit targets, there are 130 type- and size-specific operators. Conversions on 32-bit targets, for instance, convert only between 4 and 4- or 8byte floats, or widen or narrow between three sizes of integers. Some operators have only one or a few valid suffixes; for instance, the address operators ADDRL, ADDRF, and ADDRG can have only the ' $P$ ' type suffix and whatever size is the size of a pointer on the target. Back end authors need accommodate only those type- and size-specific operators that are meaningful on their targets.

Incidentally, the lcc 3.x interface [4] supported only three sizes of integers, two sizes of floats, and insisted that pointers fit in unsigned integers. These assumptions simplified the compiler and were suitable for 32-bit architectures, but not for 64-bit architectures. The main difference between the 3.x interface and the 4.x interface described here are the operator size suffixes.

Figure 4 summarizes the purpose of the 18 code-generation procedures. On most targets, implementations of many of these routines are very short, perhaps only a few calls to printf, because they simply emit assembly language. Most of the work goes into gen, emit and function, which collaborate to generate and emit code for a function. While not required by the interface, all of lcc's distributed back ends use a variant of the IBURG codegenerator generator [10] to specify instruction selection. The resulting code generators emit locally optimal code. Instruction selection specifications and target-dependent functions run about 700 lines per target. There are about another 900 lines of code that are shared between all targets and include functions for register allocation, etc.

lcc's packaging is somewhat novel: Pointers to the code-generation procedures and some target-specific parameters are packaged in the following 'interface record:'

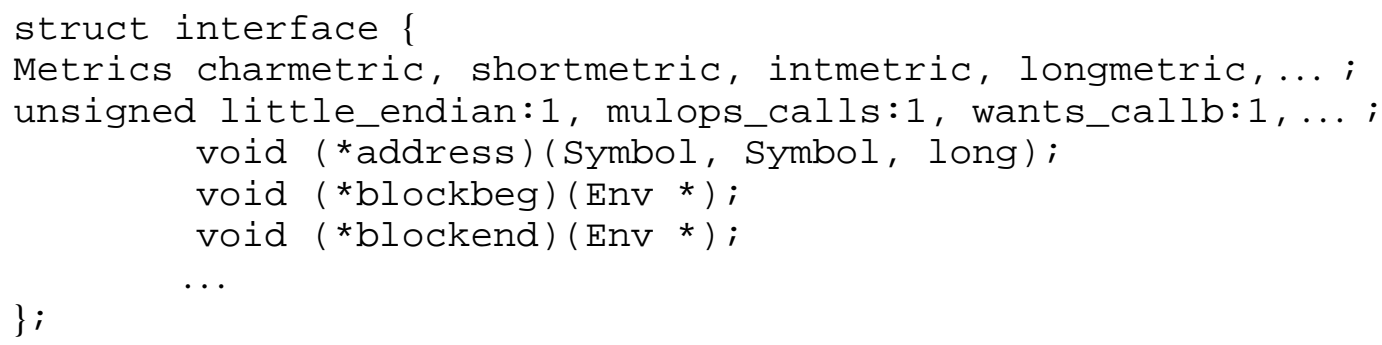

The Metrics values give the sizes and alignments of the basic data types, and the 1-bit flags identify other target-dependent features, like endianness. There is one interface record 


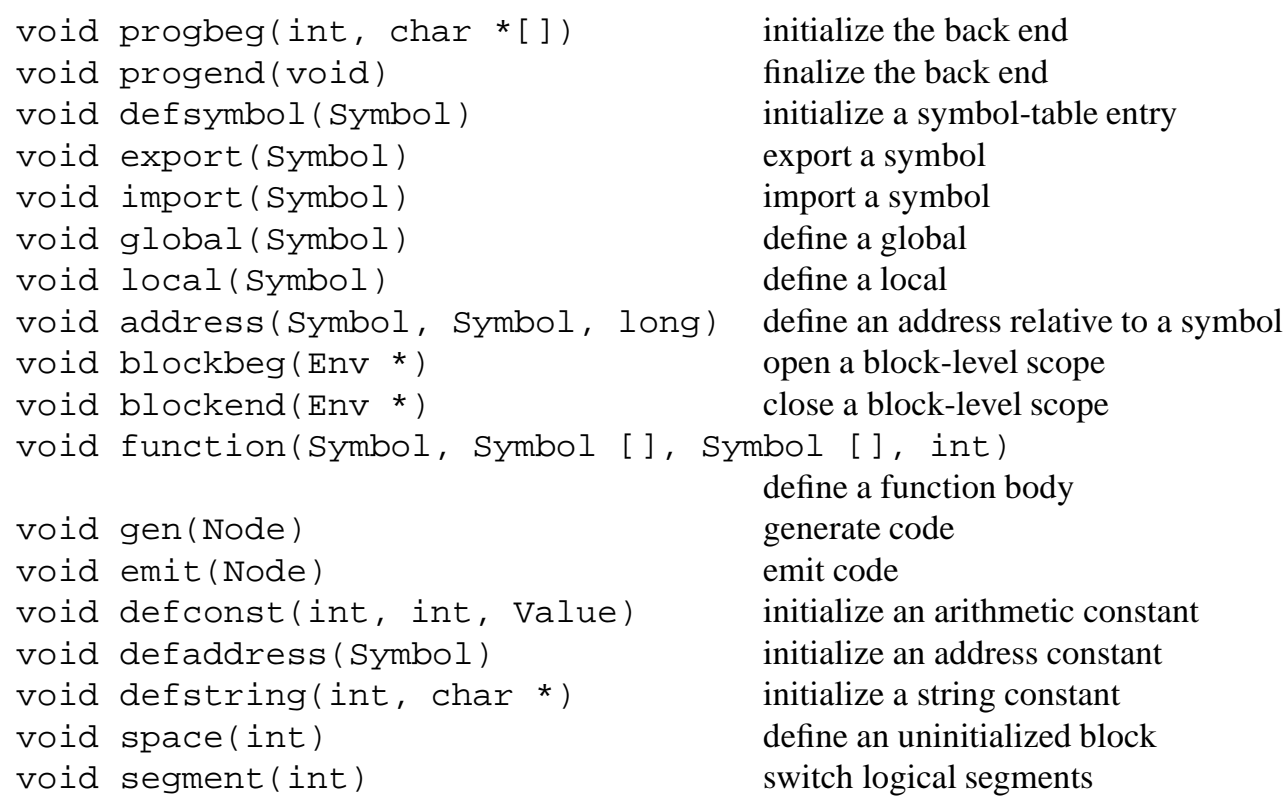

initialize the back end

finalize the back end

initialize a symbol-table entry

export a symbol

import a symbol

define a global

define a local

define an address relative to a symbol

open a block-level scope

close a block-level scope

define a function body

generate code

emit code

initialize an arithmetic constant
initialize an address constant

initialize a string constant

define an uninitialized block

switch logical segments

Figure 4. lcc code-generation procedures

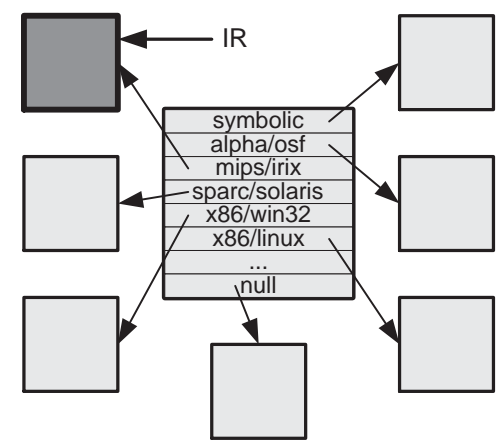

Figure 5. Specifying a target, e.g. mips/irix, points IR at an interface record

for each distinct target, but different records can share functions. lcc is a small compiler, so all of the back ends are combined into a single executable program, which makes lcc a cross compiler. As depicted in Figure 5, a command-line option selects the desired target, e.g.

lcc - Wf-target $=$ mips/irix $-S$ wfl.c

causes lcc to compile $\mathrm{w} f 1 . \mathrm{c}$ and leave the generated MIPS assembly code in wf $1 . \mathrm{s}$. The $-W f$-target option points IR to the appropriate interface record, and the front end makes indirect calls to the code-generation procedures, e.g.

$$
\text { ( *IR->defsymbol) (p) ; }
$$

The default target is the host, so this option is required only for cross compilation. 


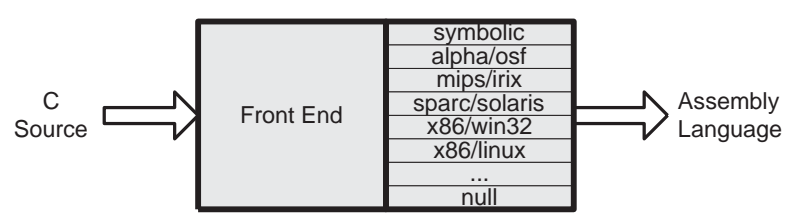

Figure 6. lcc's monolithic design: One front end, numerous back ends

\section{DIVIDING lcc}

lcc is - by design - a monolithic compiler: The front end and the back ends are combined into a single address space, so the front and back ends communicate by procedure calls that exchange pointers to shared data structures read and written by both parties, as Figure 6 illustrates. Also, back ends can make upcalls to functions provided by the front end, and the front end reads data structures written by the back ends. There are about a half dozen such functions, e.g. data-structure constructors, a memory allocator, type predicates, and so on.

lcc is small, at least in comparison with other compilers, because it omits some components, most notably a global optimizer. One way to add more functionality is to split lec into a separate front end and one or more separate back ends so that an optimizer can be run between these programs. This design would also make it easier to use lcc in research projects.

Splitting lcc into two separate programs requires either massive revisions or some way to read and write the data and actions represented by the existing code-generation interface. ASDL facilitates the second alternative: It helps divide lcc into separate programs with no change to the code-generation interface. So, the existing back ends can be used unmodified.

Figure 7 depicts this revised design. The front end, rcc, emits a pickle that encodes all the data structures and the function calls made when compiling a $\mathrm{C}$ source file. Essentially, rcc converts the lcc IR to an IR defined by the ASDL grammar. The new program, dubbed 'pass2', reads a pickle, recreates the internal data structures, and makes the function calls encoded in the pickle. That is, pass 2 converts the ASDL-defined IR back to the lcc IR. The generated assembly language is often byte-for-byte identical to the code emitted by the monolithic compiler. Differences occur only when the back end calls the label generator, which causes the revised design to number labels differently.

asdlGen reads the lcc-specific ASDL grammar described in the next section and emits $\mathrm{C}$ code for the data-structure constructors, readers, and writers, which is included in both $\mathrm{rcc}$ and pass2. Otherwise, the revised front end, rcc, is nearly identical to the original front end. The ASDL emission is accomplished by an ASDL back end, which 'spoofs' the back end by overwriting the target-specific code-generation function pointers with pointers to its own functions. The back ends are actually linked into both rcc and pass 2 , because the interface records carry important machine parameterizations required by both programs. This packaging is not essential; rcc could link in the interface records without the functions.

The revised compiler is used much like the original, except that ASDL output must be specified to rcc, and pass 2 must be run to emit the generated code, e.g.

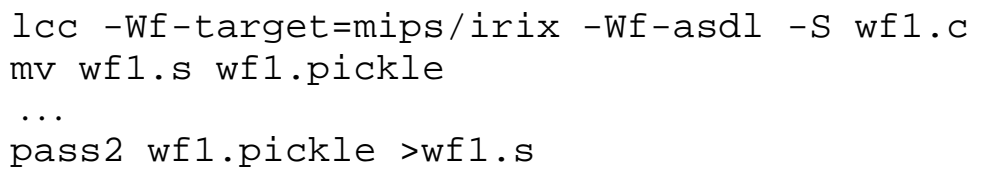




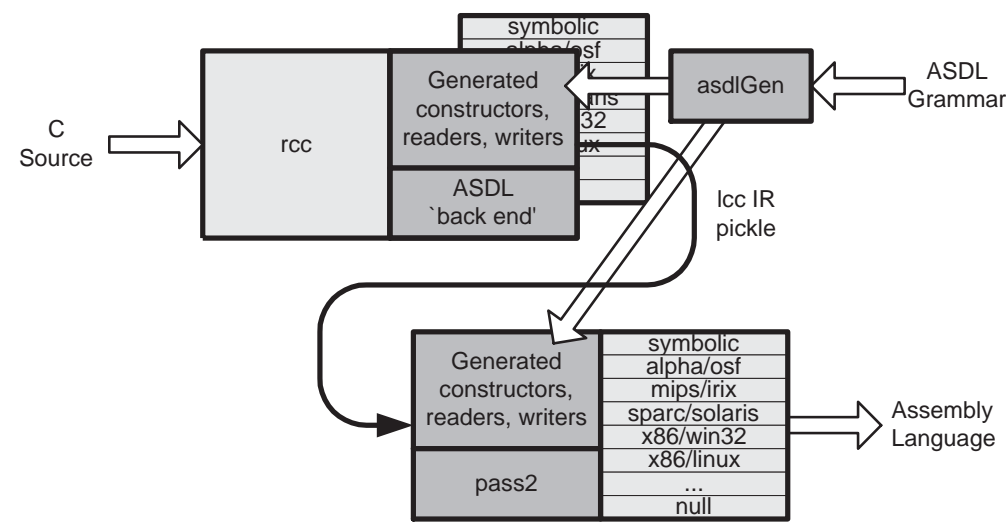

Figure 7. lcc's revised design: Separate front end and back ends

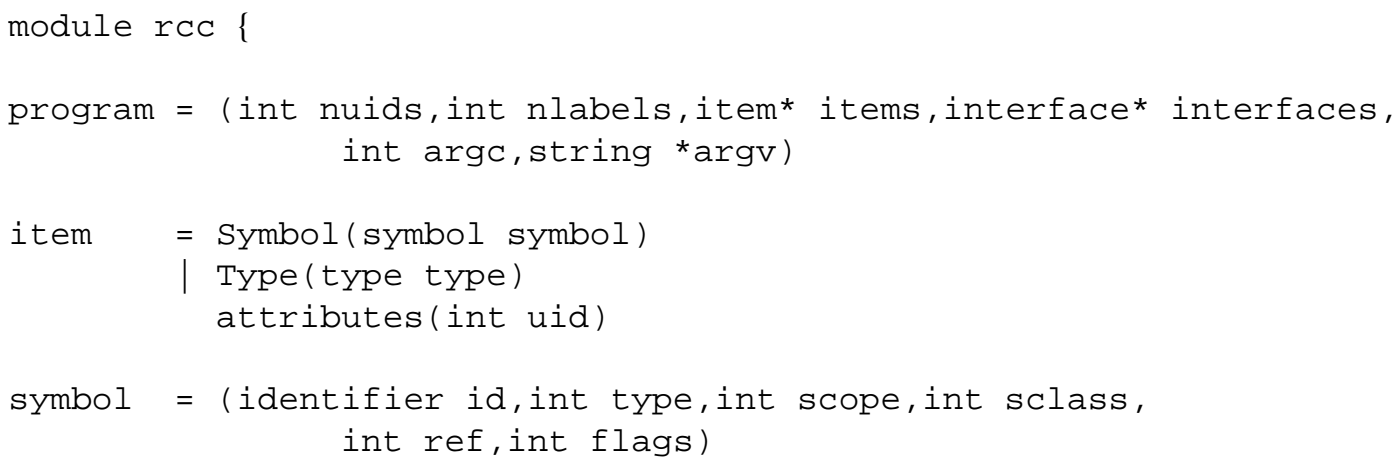

See Figure $9 \ldots$

See Figure $12 \ldots$

See Figure $10 \ldots$

\}

Figure 8. The ASDL grammar for lcc's code-generation interface

The first command runs rcc and leaves the pickle in wf1.s, which the second command renames. The third command generates the MIPS assembly code in wf $1 . \mathrm{s}$, perhaps after it has been optimized or otherwise processed by an intervening step.

\section{The ASDL grammar}

The ASDL grammar for lcc is small—only about 70 lines. It specifies data structures that are target-dependent, which means, for example, that it is impossible to specify, say, the 
mips / irix target to rcc and the $x 86 /$ win 32 target to pass 2 . Indeed, pass 2 does not accept a target option, because the target specification is embedded in the pickle.

Figures 8, 9, 10 and 12 show the complete lcc ASDL grammar. The grammar specifies more than just what is in the code-generation interface, because pass 2 must recreate the compilation environment built by rcc, the front end. There are two important ramifications of this requirement. First, the pickles include complete $\mathrm{C}$ language type information, for example, everything about structures and unions, etc. The code-generation interface includes only the six basic types. This information is defined by the type sum type. Secondly, lcc's code-generation data structures and related internal structures are graphs, not trees. Thus, items with multiple references are identified by integers, and the references to them replaced by these integers, even when these items are referenced only once. Fields named uid in the grammar identify these integers. Dealing with graphs is ASDL's major shortcoming.

The product type program is the first ASDL type in Figure 8, and an instance of this type represents a $C$ compilation unit. That is, rcc 'compiles' a $C$ source file into a program and writes it to a pickle, which pass 2 reads and traverses to generate code. A program carries counts of the number of unique integers - uids for short - and the number of generated labels, a sequence of item types, a sequence of interface types, the command-line argument count, and the arguments themselves. string is a built-in ASDL type. The sum type item carries a uid (as an attribute) and either the associated symbol or $\mathrm{C}$ type. The item sequence in a program associates uids with symbols and types, as described below.

Symbol-table entries are an example of a multiply referenced data structure. Symboltable entries are represented by the ASDL product type symbol (see Figure 8), which is a straightforward rendition of lcc's internal symbol-table entry. It carries the symbol's name, C type, scope, storage class, how often it's referenced, and some flags. For example, the C declaration

struct elem \{ int count; struct elem *left, *right; char *word; * root;

declares root to be a pointer to a 'struct elem'. The corresponding symbol is

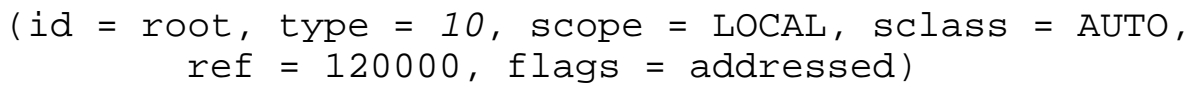

where, for clarity, symbolic values appear for the scope, sclass, and flags fields. The id field is an instance of the built-in ASDL type identifier, which are atoms. The type field- ' 10 ' in this example-is a uid that identifies a type value defined somewhere else in the item sequence. Here and below, uids are shown in a slanted typewriter font, and the displays themselves are given in an $a d$ hoc descriptive format derived from a program that prints lcc ASDL pickles. The ASDL browser [3] also displays pickles in a generic format.

\section{Types}

C language types are represented by instances of the sum type type defined in Figure 9, and are essentially abstract syntax trees of the $\mathrm{C}$ type constructors. For example, INT is a basic type; POINTER represents a pointer type and its integer field is the uid of the referent type; and STRUCT represents a structure type with a tag and an ordered set of fields. The fields are represented by a sequence of field product types, one for each field, giving the field name, its type, offset, and location information for bit fields. Other types are similarly represented. Every type has attributes that give its size and alignment constraint in bytes. 


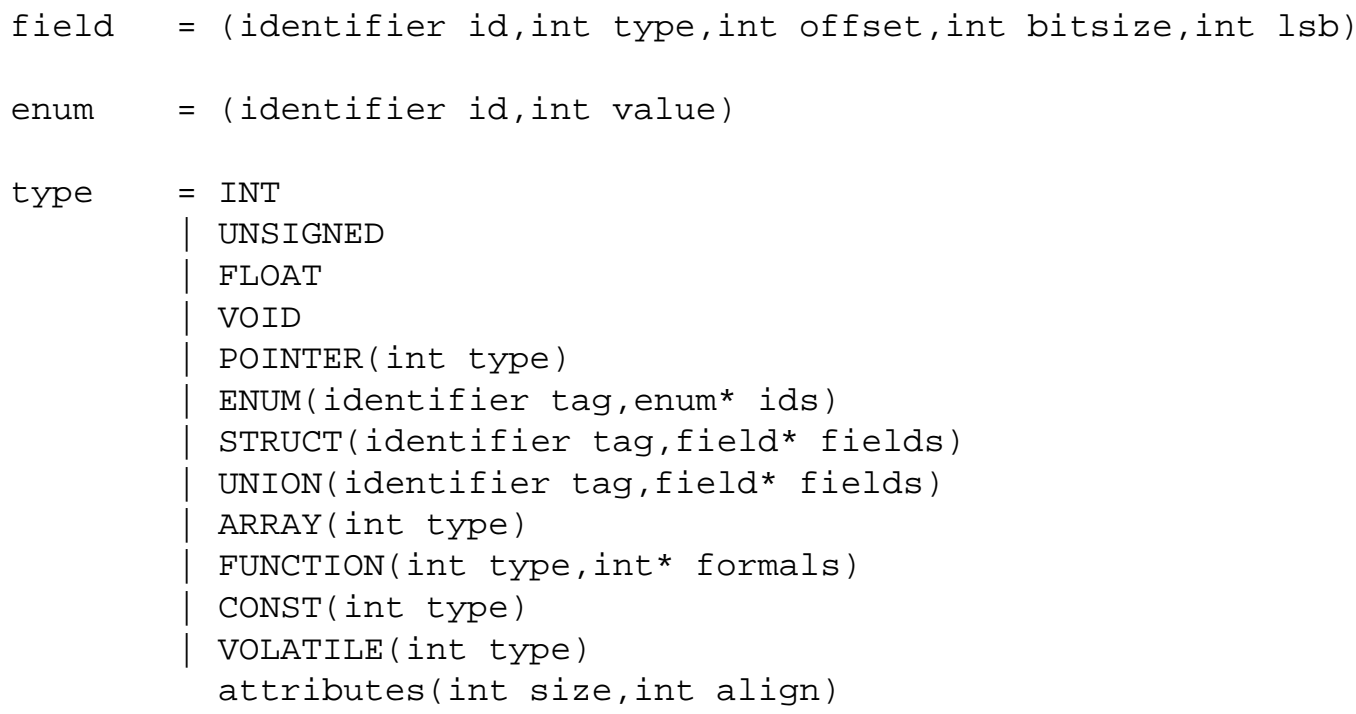

Figure 9. ASDL grammar for C types

A snippet of the item sequence for the type representing the $\mathrm{C}$ type 'struct elem' defined previously helps clarify the definition of uids and their use:

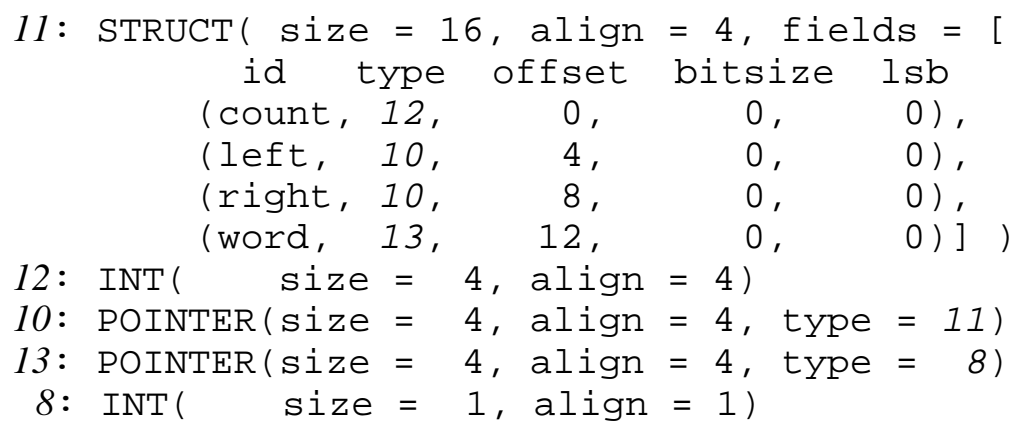

Again, the italicized numbers are uids. The uids on the left are the uid attributes in the item type, and each of these define a uid and its associated type. The occurrences of uids in a type field are references to types. Type 11 is the type value for 'struct elem'; its fields give the size of instances of this struct (16 bytes), their alignment (on 4-byte boundaries), and their fields. Each of the field values in the sequence include a uid for the type of that field. Type 10 is the $\mathrm{C}$ type 'struct elem *'. Notice the two kinds of INTs: Type 12 is a 4-byte integer, which is the $C$ type 'int', and type 8 is a 1-byte integer, which is type $C$ type 'signed char'. Thus, type 13 is the $\mathrm{C}$ type 'char*'.

\section{IR trees}

IR trees are represented by a nearly isomorphic set of trees defined by the ASDL sum type node, defined in Figure 10. Some generic operators are represented by corresponding constructors, e.g. CNST and ADDRL. Others are represented by constructors for a class of 
node

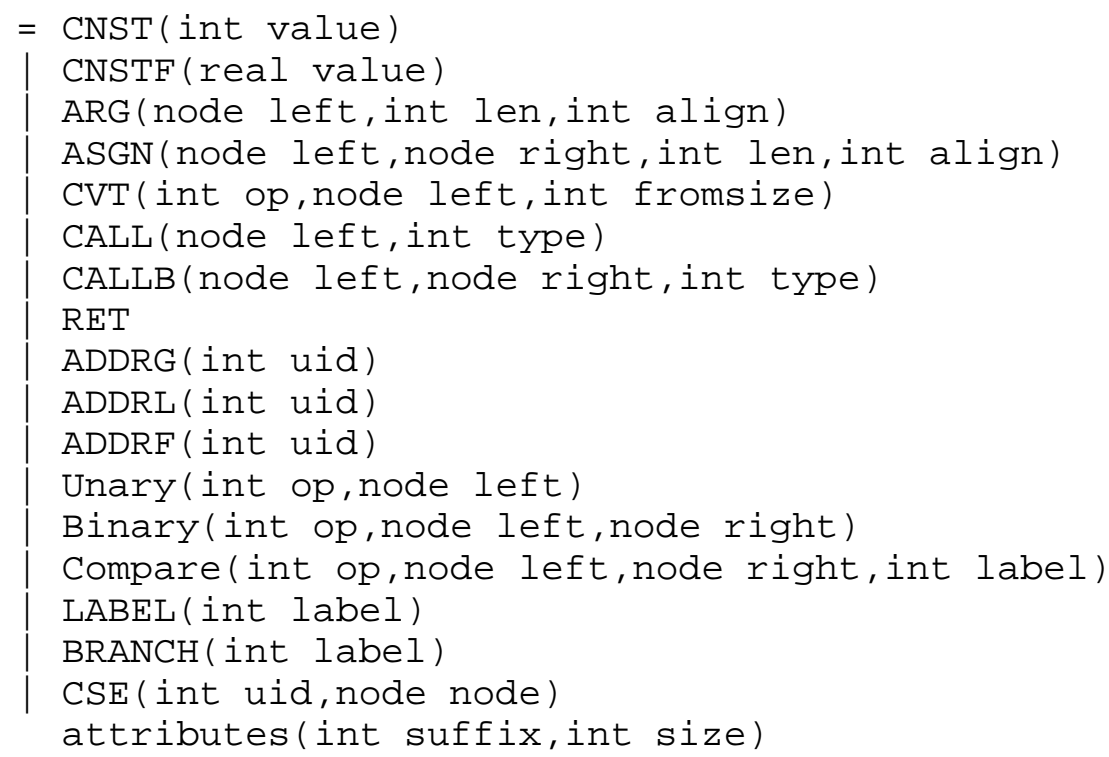

Figure 10. ASDL grammar for IR trees

generic operators in which the specific operator is provided as a parameter: CVT nodes represent the conversion operators (CVF, CVI, CVP and CVU), Unary and Binary nodes represent the unary (INDIR, RET, JUMP, NEG, BCOM) and binary operators (ADD, SUB, DIV, MUL, MOD, BOR, BAND, BXOR, RSH, LSH), and Compare nodes represent the comparisons $(\mathrm{EQ}, \mathrm{NE}, \mathrm{GT}, \mathrm{GE}, \mathrm{LE}, \mathrm{LT})$. The op field in these kinds of nodes holds the appropriate lcc operator.

ADDRL, ADDRP and ADDRG nodes address locals, parameters, and globals; the uid values identify the appropriate symbol-table entries. LABEL nodes are label definitions, and BRANCH nodes are unconditional jumps. Compare, LABEL, and BRANCH nodes use label numbers instead of symbol-table entries for labels; pass 2 recreates the symbol-table entries as it reconstructs the IR. CSE nodes identify common subexpressions and associate a symboltable entry for a temporary ( $u i d)$ with a node that computes the subexpression (node); subsequent uses of the subexpression are given by fetching the value of the temporary (with IND IR and ADDRL nodes). Every node includes suffix and size attributes, which correspond to the type and size suffixes in the type- and size-specific IR operators.

It is important to realize that nodes are not lcc IR trees-they represent IR trees. In pass2, nodes provide the data necessary to recreate the lcc IR trees, which are passed to the back ends. This 'duplication of effort' is an onerous side effect of retrofitting an existing compiler with ASDL and is discussed in more detail below.

lcc compiles the $\mathrm{C}$ code

char * $\mathrm{S}$; int $\mathrm{C} ;{ }^{*} \mathrm{~S}++=\mathrm{C}$;

into the equivalent of

$t 1=s ; s=t 1+1 ; \star t 1=c ;$

where $t 1$ is a compiler-generated temporary. Figure 11 shows the ASDL nodes for these three statements on a 32-bit target. The notation ASGN P 4 gives the constructor, the suffix 

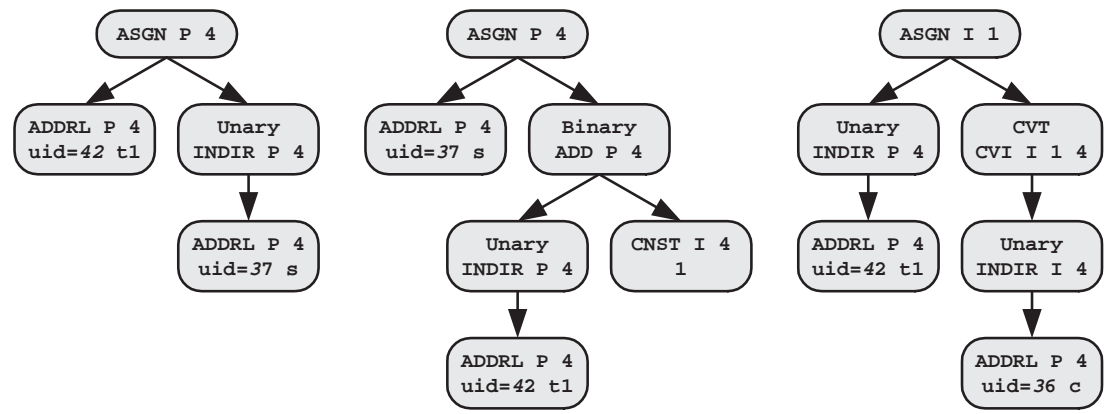

Figure 11. ASDL representation for $\mathrm{t} 1=\mathrm{s} ; \mathrm{s}=\mathrm{t} 1+1 ; \star t 1=\mathrm{c}$

attribute as one of the types listed above, and the size attribute. Notice the constructor for the indirection in the leftmost tree; it's a Unary node with three values: operator INDIR, suffix $\mathrm{P}$, and size 4. The node for Binary is similar. Leaves, like ADDRL, include the uid of the appropriate symbol. For clarity, Figure 11 shows the names, too, e.g. $t 1$, but the names are not in the node.

Nodes (and all ASDL-defined data structures) are written to pickles in a compact, prefix, binary representation in which integers can take as little as one byte. For example, the leftmost tree in Figure 11 takes 15 bytes:

ASGN P 4 ADDRL P 442 Unary INDIR P 4 ADDRL P 437

\section{Interface calls}

The ASDL types described above encode the data structures in lcc's code-generation interface. The ASDL sum type interface, defined in Figure 12, encodes the calls made from the front end to the back end. Compare this type definition with the interface calls listed in Figure 4. The only significant change is that symbol-table pointers have been replaced by the corresponding uids or sequences of uids. progbeg and progend have been omitted, because pass 2 can simply make these calls; pass 2 also supplies the actual arguments for blockbeg and blockend, so these arguments are not included in interface. Calls to defconst with real values are represented by a separate constructor, Defconst $f$, which confines the use of real values. ASDL has no built-in support for reals, so they are represented with integers for their most significant and least significant bits.

Address and Local constructors associate uids with 'relative' symbols and with locals and parameters. A relative symbol is one that is defined by a constant offset from another symbol, e.g. a [i+10] would elicit a definition of a symbol to represent the address of $\mathrm{a}[10]$, which is known at compile time. Instances of Forest carry the nodes that represent the executable code in each function. These appear in the interface list in the codelist field of functions. There is one function for each $\mathrm{C}$ function in the input.

Here's an example: The small function

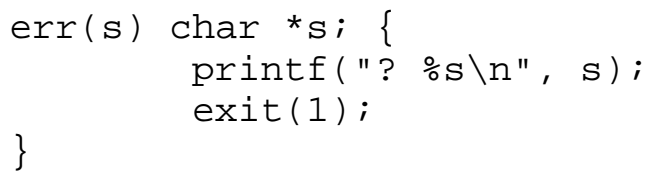


real

interface
$=($ int msb, int lsb)

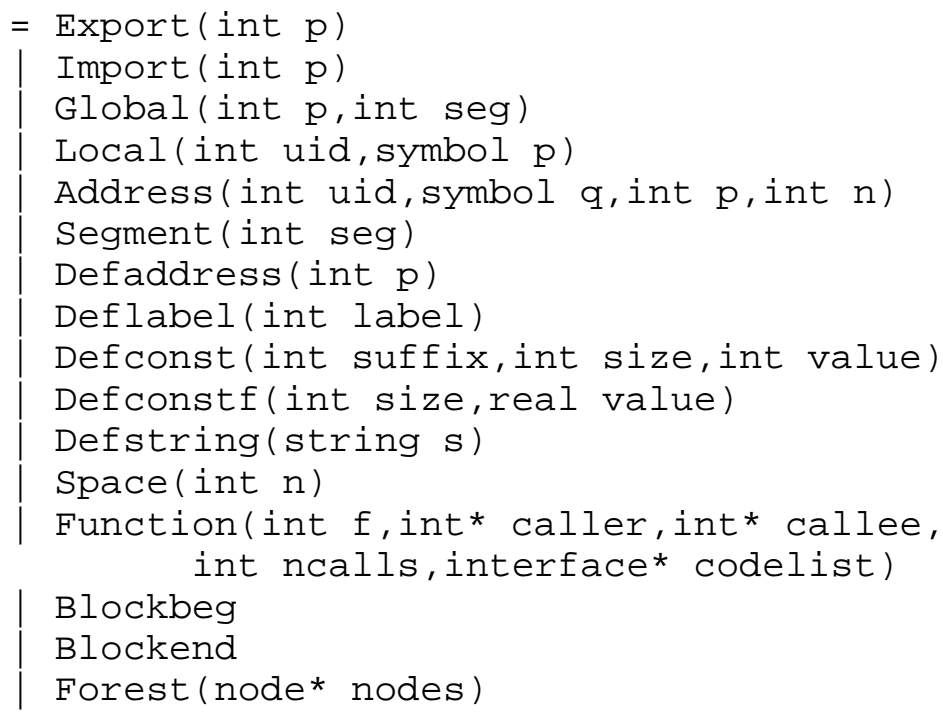

Figure 12. ASDL grammar for code-generation interface calls

yields the following sequence of interfaces:

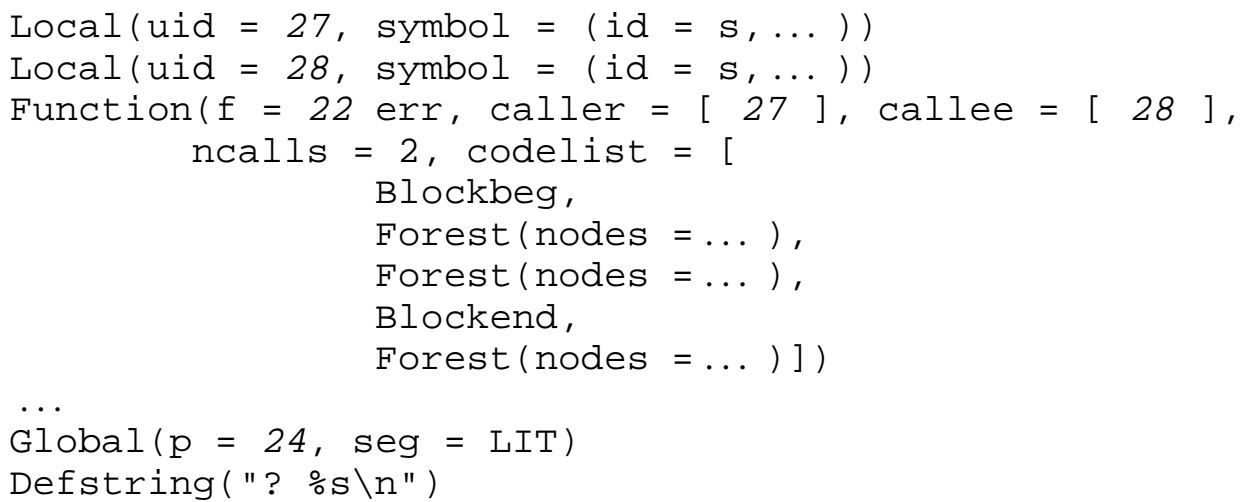

The Locals above define two views of the formal parameter $s$, one as seen by callers of err and one as seen by the callee itself. These have the same name- $s-$ but are different symbols, as indicated by their different uids. Often, these symbols are identical, but there are important cases when they are different, as detailed below. The first two occurrences of Forest carry the nodes for the two function calls. The third Forest holds a single LABEL node that marks the location of the function epilogue. Global and Defstring collaborate to initialize a compiler-generated static variable for the format string shown.

\section{MEASUREMENTS}

While retrofitting lcc to use ASDL changed lcc's structure dramatically, this process did not add much code. The ASDL grammar described in the previous section is about 70 lines, the 
ASDL back end is 409 lines of $\mathrm{C}$, and pass 2 is 681 lines of C. Most of the three months this project consumed was devoted to revising the grammar and adapting the ASDL back end and pass 2 to these revisions. This code is available in lcc 4.1.

The 70-line ASDL grammar generates about 2183 lines of $\mathrm{C}$ declarations and function definitions. This code is approximately what would be required if the ASDL-generated constructors, readers, writers were written by hand. The savings would increase if the ASDL grammar were used to generate code in other languages. For example, if an optimizer were written in Java, it would use the 3332 lines of Java generated from the same ASDL grammar.

On Windows NT, the size of the monolithic compiler executable is $380 \mathrm{~KB}$ (produced by the Microsoft Visual C/C++ 5.0 compiler with $-\mathrm{O} 1$ optimization). The revised rcc with the ASDL back end, and the generated constructors, readers and writers is $437 \mathrm{~KB}$, and pass 2 is $395 \mathrm{~KB}$. Rcc includes the back ends for all of lcc's targets, because that packaging is the simplest one. The code for these back ends and the symbol-table emission code could be omitted saving about $199 \mathrm{~KB}$.

Table I summarizes the compilation times and the file sizes for the monolithic and divided variants of lcc when compiling its own non-trivial modules. The times are given in centiseconds and are for the compilation phase only; that is, the timings do not include preprocessor and assembler times. All timings were taken on a lightly loaded $200 \mathrm{MHz}$ Gateway PC with $128 \mathrm{MB}$ of RAM and SCSI disks running Windows NT 4.0. The compiler variants were compiled by the Microsoft Visual $\mathrm{C} / \mathrm{C}++5.0$ compiler with $-\mathrm{O} 1$ optimization.

The first column gives the time in centiseconds for compiling the module named in the rightmost column with the monolithic version of lcc. The second and third columns give the compilation times for rcc and pass 2 . Thus, for example, the fourth row shows that the monolithic compiler compiled dag . $\mathrm{c}$ to assembly language in 33 csecs., and rcc and pass 2 accomplished the same task in $61+31=92$ csecs.

The fourth column gives the size of each module's pickle in kilobytes. By way of comparison, the fifth column gives the size of the corresponding unoptimized object file produced by the Microsoft Visual $\mathrm{C} / \mathrm{C}++$ compiler. Pickles contain complete symbol-table information, so perhaps a more meaningful comparison is with the sizes of object files with embedded symbol tables, which the sixth column shows.

Input/output time dominates the compilation times. The revised rcc is about 1.5-2 times slower than the monolithic compiler; building the ASDL data structures and emitting them accounts for most of this time. As detailed in the next section, rec essentially duplicates its data structures as it builds the ASDL representation, which costs both time and space.

pass 2 is faster than both the monolithic compiler and rcc because it doesn't have to read and analyze the $\mathrm{C}$ source code; it simply inhales the pickle, rebuilds the compiler's data structures, and calls the back end functions. While rcc plus pass 2 adds a factor of 2-3 to the compilation time, lcc is fast enough that this overhead is acceptable, especially in an experimental setting; for example, the monolithic compiler compiles itself in 15 secs., and rcc plus pass 2 takes 35 secs.

Pickle sizes run about 3 times the sizes of object files and about twice the sizes of object files with embedded symbol tables. Compression could reduce pickle sizes to that of object files; for example, compressing all of the pickles listed in Table I yields a $867 \mathrm{~KB}$ zip file. Each pickle includes the symbol-table entries from the common header files included by each module. Pickle sizes could also be reduced by emitting these symbol-table data into a separate pickle and omitting them from the per-module pickles. 
Table I. Compilation times and output file sizes.

\begin{tabular}{|c|c|c|c|c|c|c|}
\hline $\begin{array}{c}\text { ICC }-S \\
100 \text { ths sec. }\end{array}$ & $\begin{array}{c}\operatorname{lcc}-S-a s d l \\
100 \text { ths sec. }\end{array}$ & $\begin{array}{c}\text { pass } 2 \\
100 \text { ths sec. }\end{array}$ & $\begin{array}{c}\text { Pickle } \\
\text { size in KB }\end{array}$ & $\begin{array}{l}\text { Object file } \\
\text { size in KB }\end{array}$ & $\begin{array}{c}\text { Object file } \\
\text { w/symbols in KB }\end{array}$ & File \\
\hline 8 & 20 & 6 & 22.2 & 1.7 & 3.4 & alloc.c \\
\hline 115 & 211 & 98 & 242.1 & 91.5 & 124.4 & alpha.c \\
\hline 15 & 33 & 13 & 37.7 & 11.8 & 24.1 & bytecode.c \\
\hline 33 & 61 & 31 & 81.7 & 26.0 & 45.4 & $\operatorname{dag} \cdot \mathrm{c}$ \\
\hline 45 & 67 & 35 & 90.5 & 33.9 & 48.5 & dagcheck.c \\
\hline 42 & 77 & 44 & 100.8 & 36.9 & 61.4 & decl.c \\
\hline 35 & 65 & 34 & 82.6 & 20.6 & 36.7 & enode.c \\
\hline 26 & 25 & 8 & 27.4 & 5.3 & 12.2 & error.c \\
\hline 48 & 65 & 34 & 81.2 & 25.3 & 44.2 & expr.c \\
\hline 44 & 67 & 33 & 77.0 & 23.7 & 47.0 & gen.c \\
\hline 24 & 40 & 20 & 40.5 & 8.4 & 20.0 & init.c \\
\hline 16 & 26 & 8 & 26.5 & 4.9 & 11.2 & input.c \\
\hline 28 & 43 & 21 & 58.9 & 20.2 & 30.9 & lex.c \\
\hline 22 & 32 & 12 & 35.7 & 8.8 & 19.8 & main.c \\
\hline 119 & 161 & 87 & 206.2 & 75.6 & 106.4 & mips.c \\
\hline 35 & 32 & 10 & 33.1 & 7.5 & 14.7 & output.c \\
\hline 18 & 28 & 11 & 34.7 & 7.1 & 18.5 & prof.c \\
\hline 19 & 33 & 13 & 34.4 & 5.3 & 11.6 & profio.c \\
\hline 55 & 69 & 30 & 82.6 & 26.8 & 69.1 & $\mathrm{rcc} \cdot \mathrm{C}$ \\
\hline 45 & 65 & 34 & 92.2 & 22.4 & 37.3 & simp.c \\
\hline 147 & 221 & 157 & 264.9 & 94.1 & 129.9 & sparc.c \\
\hline 44 & 35 & 13 & 40.1 & 11.0 & 22.9 & stab.c \\
\hline 40 & 52 & 25 & 63.5 & 20.1 & 39.6 & stmt. c \\
\hline 16 & 28 & 8 & 25.5 & 2.7 & 4.8 & string.c \\
\hline 25 & 31 & 11 & 35.6 & 11.8 & 26.2 & sym.c \\
\hline 45 & 51 & 18 & 55.1 & 22.7 & 43.0 & symbolic.c \\
\hline 17 & 32 & 12 & 32.4 & 8.0 & 18.5 & trace.c \\
\hline 44 & 32 & 12 & 35.2 & 7.6 & 15.1 & tree.c \\
\hline 40 & 59 & 31 & 79.7 & 28.9 & 49.5 & types.c \\
\hline 185 & 249 & 177 & 298.0 & 97.3 & 133.9 & $\times 86 . c$ \\
\hline 182 & 342 & 227 & 365.5 & 117.1 & 157.9 & x86linux.c \\
\hline 1577 & 2352 & 1150 & 2783.5 & 885.0 & 1428.1 & Total \\
\hline
\end{tabular}

\section{EVALUATION}

Retrofitting lcc to use ASDL highlighted some strengths and weaknesses in both ASDL and lcc. One of the somewhat unexpected strengths of ASDL is that it helps find bugs. Writing the ASDL back end revealed two related long-standing bugs in lcc. The first is illustrated by the following code.

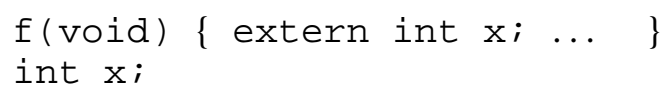

The two declarations for $x$ refer to the same identifier. The error is that lcc created two symbol-table entries for $\mathrm{x}$ : One was created at the extern declaration for $\mathrm{x}$ and used when compiling the body of $f$, and the other one was created at the top-level declaration for $\mathrm{x}$ and used thereafter, including announcing the definition of $\mathrm{x}$ via the code-generation function global (see Figure 4). It was intended that there be only one symbol-table entry for $\mathrm{x}$. 
These symbol-table entries had identical contents, and all of the existing back ends examined only the contents. The ASDL back end, however, used the pointer to the symbol-table entry as a handle to the corresponding ASDL symbol type (see Figure 8), and thus erroneously created two symbols. References to $x$ from within $f$ referred to the wrong symbol and thus the generated code was incorrect - it was if the code had been written as

$f($ void) $\{$ static int $x ; \ldots$ \}

int $x$;

The second bug adds another twist to the first bug and is illustrated by the following code.

static int $x$;

$\mathrm{f}$ (void) $\{$ extern int $\mathrm{x} ; \ldots$ \}

Again, lcc erroneously created two symbol-table entries when one was expected. It also changed the storage class of the $x$ declared within $f$ to be static when it was announced to the back end, then changed it back to extern. As a result, the $x$ appeared to be static when declared and extern when used. On targets that handle statics differently than externs, pass 2 emitted incorrect code.

ASDL exposed some awkward binding times in the lcc code-generation interface, which required revising the implementation. lcc compiles the function

$f(x, y)$ char $x$; int $y ;\{\ldots\}$

as

f $\left(?\right.$ int $x^{\prime}, ?$ int $\left.y^{\prime}\right)\left\{\right.$ ? char $\mathrm{x}=x^{\prime}$; ? int $\left.\mathrm{y}=y^{\prime} ; \ldots\right\}$

lcc generates two symbol-table entries for each parameter: one for the parameter as passed by the caller- $x^{\prime}$ and $y^{\prime}$ in the code above - and one for the parameter as seen by the callee$x$ and $y$ above. It generates assignments of the caller parameter to the corresponding callee parameter if their types differ or if their storage classes differ. In the example above, in which the occurrences of ? denote storage classes, the char parameter $\mathrm{x}$ is promoted and passed as an int, so the types of $\mathrm{x}$ and $x^{\prime}$ differ. Back ends can change the storage class of caller and callee parameters to reflect target-dependent calling conventions. On the MIPS, for example, $y^{\prime}$ is passed in a register, so an assignment to $\mathrm{y}$ is generated if $\mathrm{y}$ lands in memory.

The binding time problem is that rcc doesn't have the information necessary to determine whether or not to generate these assignments. Storage class information is known only to the back end and thus isn't known until pass 2 runs. The solution was to move the code that generates these assignments into pass 2 .

A similar problem arises in common subexpression elimination (CSE), but requires extra work by both rcc and pass2. lcc does CSE on extended basic blocks, but it needs to know something about register assignments before it hoists an rvalue into a temporary. For example, in the expression

$\mathrm{a}=\mathrm{b} * \mathrm{c}+\mathrm{b} * \mathrm{~d}$

the rvalue of $b$ is a common subexpression. lcc copies $b$ to a temporary, but only if the temporary is in a register and $b$ isn't. Again, rcc does not have the data necessary to make that decision, because the back end has the final say on storage classes. So, rcc makes a conservative assumption and generates temporaries for all multiply referenced rvalues, and pass2 eliminates those that don't pay. 
These binding-time problems would have been exposed during any attempt to partition lcc. Earlier attempts to divide lcc, however, could get away with simple, problem-specific mechanisms to avoid changing the front-end implementation [11].

One of the flaws in ASDL is that it can lead to a duplication of data structures, which perhaps should be expected when modifying an existing compiler to use ASDL. lcc builds numerous data structures to represent the $\mathrm{C}$ source program, e.g. symbol-table entries, tree nodes, strings, etc. Most of the code in the ASDL back end is devoted to building copies of these data structures - that is, building a different, but logically equivalent representation for nearly everything. All this duplication could be avoided if ASDL were used at the outset to define all the important data structures, but this approach would have required a much more drastic revision of lcc.

Perhaps the biggest nuisance in using ASDL is dealing with non-tree data structures, e.g. by using uids for symbols and types. These are common and there should be a better way to handle them, or at least some more built-in support for defining and referencing them.

The lcc ASDL grammar is 'ambiguous'; that is, it permits construction of type instances that do not represent valid lcc code-generation interface structures. For example, the grammar in Figure 12 permits a Function whose codelist field includes another Function. This sequence of calls never occurs in lcc. Similar comments apply to the CVT, Binary, Unary, and Compare constructors: any lcc operator could be given as the op field. Ambiguity shortens grammars, much the same way as an ambiguous YACC grammar is smaller than a non-ambiguous one. While few bugs could be attributed directly to using an ambiguous grammar, the savings probably isn't worth it. A non-ambiguous ASDL grammar, which might be no more than 50 per cent longer, would catch more errors at compile-time and it would document the semantics more accurately.

\section{CONCLUSIONS}

Revising lcc to use ASDL was, overall, straightforward, and the resulting components-rcc and pass 2-provide an improved platform for compiler-related research using lcc. It is now possible to insert additional passes into the compilation pipeline without modifying or even understanding the front and back ends. The obvious first candidate is a global optimization pass. Adding an optimizer will surely identify weaknesses in the current ASDL grammar. For example, it is likely that additional data structures, such as a flow graphs, will be needed. The optimizer could build a flow graph itself, but it may prove useful to add these kinds of generally useful structures to the pickles.

Fortunately, ASDL can accommodate additions gracefully. A pickle consists of one or more instances of ASDL types. Currently, lcc pickles hold just an instance of program defined in Figure 8. Other passes can append instances of additional types to pickles and use these instances without affecting pass2, because pass 2 reads only the instance of program.

The experience with lcc suggests that ASDL would be useful in similar projects. It might be even more useful in new compilers and related projects, because using ASDL would provide concise documentation of the important data structures and would generate the code for constructing, reading, and writing them. Using ASDL from the start would avoid the duplication of data structures and of effort described in the previous section. Early use of ASDL might encourage the creation of a multi-language library of general-purpose functions for manipulating ASDL-defined structures.

ASDL is equivalent to Document Type Declarations (DTDs) in XML [12], so it is natural to wonder if the increasing investment in XML tools can be leveraged to provide better compiler 
infrastructure tools. As a first step, the ASDL pickle readers and writers have been modified to emit pickles in XML instead of in the original binary format. These pickles are necessarily huge, because they are written in readable ASCII, but they compress to approximately the sizes suggested in Table I; for example, the XML pickles for all of the modules listed in Table I compress into a $1394 \mathrm{~KB}$ zip file. They can, however, be examined and processed by generic XML browsers and editors, which obviates the need for ASDL-specific tools.

Work is also underway on the minimal support for non-tree data structures. XML supports ID and IDREF 'attributes;' these provide a way to name an instance of a type and to refer to it from instances of other types, which is essentially identical to the use of uids in the lcc ASDL grammar. Similar features may be added to ASDL.

Finally, ASDL is an ideal way to specify abstract data types and application programming interfaces (API), independent of whether or not they are going to be pickled. ASDL grammars are compact, language-independent, and hide implementation details. Debugging an ASDL grammar is usually much easier than debugging the corresponding handwritten code. With sufficient care, ASDL grammars might help simplify both the implementations of APIs and their tedious language-specific descriptions.

\section{ACKNOWLEDGEMENTS}

Daniel C. Wang wrote asdlGen and responded promptly when rec and pass2 exposed bugs. $\mathrm{He}$ also wrote the XML pickler mentioned in the last section.

\section{REFERENCES}

1. Standard Performance Evaluation Corp., Manassas, VA, SPEC, 1996.

2. H. Kienle and U. Hölzle, 'Introduction to the SUIF 2.0 compiler system', Technical Report TRCS97-22, Computer Science Department, University of California at Santa Barbara, Santa Barbara, CA, December 1997.

3. D. C. Wang, A. W. Appel, J. L. Korn and C. S. Serra, 'The Zephyr abstract syntax description language', Proceedings of the Conference on Domain-Specific Languages, Santa Barbara, October 1997, pp. 213-227.

4. C. W. Fraser and D. R. Hanson, A Retargetable C Compiler: Design and Implementation, Addison-Wesley, Menlo Park, CA, 1995.

5. S. P. Harbison and G. L. Steele, Jr., C: A Reference Manual, Prentice Hall, Englewood Cliffs, NJ, 1995.

6. T. B. Steel, Jr., 'A first version of UNCOL', Proceedings Western Joint Computer Conference, May 1961, pp. 371-378.

7. The Open Group, Cambridge, MA, Architecture Neutral Distribution Format (XANDF) Specification, January 1996.

8. R. T. Snodgrass, The Interface Description Language: Definition and Use, Computer Science Press, Rockville, MD, 1989.

9. D. R. Hanson, C Interfaces and Implementations: Techniques for Creating Reusable Software, AddisonWesley, Reading, MA, 1997.

10. C. W. Fraser, D. R. Hanson and T. A. Proebsting, 'Engineering a simple, efficient code-generator generator', ACM Letters on Programming Languages and Systems, 1(3), 213-226 (1992).

11. M. F. Fernandez, 'Simple and effective link-time optimization of Modula-3 programs', Proceedings of the ACM SIGPLAN'95 Conference on Programming Language Design and Implementation, La Jolla, CA, June 1995, pp. 103-115.

12. C. F. Goldfarb and P. Prescod, The XML Handbook, Prentice Hall, Englewood Cliffs, NJ, 1998. 